\title{
A THEOREM ON $C$-TOTALLY REAL MINIMAL SURFACE
}

\author{
SEIICHI YAMAGUCHI, MASAHIRO KON AND YASUSHI MIYAHARA
}

\begin{abstract}
We prove the corresponding theorem for a $C$-totally real minimal surface to Yau's theorem for a totally real minimal surface of a Kähler surface with constant holomorphic sectional curvature.
\end{abstract}

1. Introduction. Recently, S. T. Yau [11] has dealt with a totally real minimal surface and proved

THEOREM A. Let $M$ be a totally real minimal surface of a Kähler surface with constant holomorphic sectional curvature $C$. Then:

(i) If $M$ has genus zero, $M$ is the standard embedding of $R P^{2}$ in $C P^{2}$.

(ii) If $M$ is a complete nonnegative curved surface, $M$ is totally geodesic or flat.

(iii) If $M$ is complete nonpositive curved with Gauss curvature $K$ and $C / 4-K$ $\geqslant \alpha>0$ for some constant $\alpha$, then $M$ is totally geodesic or flat.

On the other hand [9], [10], we have introduced the notion of a $C$-totally real submanifold of a Sasakian manifold and proved

TheOREM B [10]. Let $\bar{M}^{2 n+1}(k)$ be a $(2 n+1)$-dimensional Sasakian manifold with constant $\phi$-holomorphic sectional curvature $k$ and $M$ be an $n$-dimensional compact C-totally real minimal submanifold of $\bar{M}^{2 n+1}(k)$. If $\|A\|^{2}<n(n+1)$ $\cdot(k+3) / 4(2 n-1)$ or, equivalently, $\rho>n^{2}(n-2)(k+3) / 2(2 n-1)$, then $M$ is totally geodesic, where $\rho$ is the scalar curvature.

The purpose of this paper is to obtain the corresponding theorem for a $C$ totally real minimal surface to Theorem $A$, that is, we shall show

THEOREM. Let $M$ be a $C$-totally real minimal surface of a 5-dimensional Sasakian manifold $\bar{M}(k)$ with constant $\phi$-holomorphic sectional curvature $k$. Then:

(i) If $M$ has genus zero, $M$ is totally geodesic.

(ii) If $M$ is a complete nonnegative curved surface, $M$ is totally geodesic or flat.

(iii) If $M$ is complete nonpositive curved with Gauss curvature $K$ and if $(k+3) / 4-K \geqslant \alpha>0$ for some constant $\alpha$, then $M$ is flat.

Received by the editors August 21, 1974 and, in revised form, November 1, 1974.

AMS (MOS) subject classifications (1970). Primary 53C54, 53A10, 53C25.

Key words and phrases. Totally real, $C$-totally real, minimal surface, a space of constant $\phi$ holomorphic sectional curvature, second fundamental form, totally geodesic. 
COROLlary. Let $M$ be a C-totally real minimal surface of a 5-dimensional unit sphere $S^{5}$. Then we have (i) and (ii) in Theorem and (iii) in Theorem can be rewritten as follows: (iii)' If $M$ is a complete nonpositive curved surface, then $M$ is flat.

2. Preliminaries. Let $\bar{M}$ be a Riemannian manifold of dimension $n+p$ and $M$ an $n$-dimensional submanifold of $\bar{M}$. Let $\langle$,$\rangle be the metric tensor field$ on $\bar{M}$ as well as the metric induced on $M$. We denote by $\bar{\nabla}$ the covariant differentiation in $\bar{M}$ and by $\nabla$ the covariant differentiation in $M$ determined by the induced metric on $M$. Let $\mathfrak{X}(\bar{M})$ (resp. $\mathfrak{X}(M)$ ) be the Lie algebra of vector fields on $\bar{M}$ (resp. $M$ ) and $\mathfrak{X}^{\perp}(M)$ the set of all vector fields normal to $M$.

The Gauss-Weingarten formulas are given by

$$
\begin{aligned}
& \bar{\nabla}_{X} Y=\nabla_{X} Y+B(X, Y), \quad \bar{\nabla}_{X} N=-A^{N}(X)+D_{X} N, \\
& X, Y \in \mathfrak{X}(M), N \in \mathfrak{X}^{\perp}(M),
\end{aligned}
$$

where $D$ is the normal connection in the normal bundle. Both $A$ and $B$ are called the second fundamental form and they satisfy

$$
\left\langle A^{N}(X), Y\right\rangle=\langle B(X, Y), N\rangle \text {. }
$$

The Gauss equation is given by

$$
\begin{aligned}
\langle\bar{R}(Z, Y) X, W\rangle= & \langle R(Z, Y) X, W\rangle+\langle B(Y, X), B(Z, W)\rangle \\
& -\langle B(X, Z), B(Y, W)\rangle, \quad W, X, Y, Z \in \mathfrak{X}(M) .
\end{aligned}
$$

Now we define the covariant derivative of second fundamental form $B$ as follows:

$$
\tilde{\nabla}_{X}(B)(Y, Z)=D_{X}(B(Y, Z))-B\left(\nabla_{X} Y, Z\right)-B\left(Y, \nabla_{X} Z\right)
$$

for any vector fields $X, Y, Z, \in \mathfrak{X}(M)$. For the second fundamental form $A$, we define its covariant derivative by setting

$$
\begin{array}{r}
\nabla_{X}(A)^{N}(Y)=\nabla_{X}\left(A^{N}(Y)\right)-A^{D_{X} N}(Y)-A^{N}\left(\nabla_{X} Y\right), \\
X, Y, \in \mathfrak{X}(M), N \in \mathfrak{X}^{\perp}(M) .
\end{array}
$$

Clearly we see $\left\langle\tilde{\nabla}_{X}(B)(Y, Z), N\right\rangle=\left\langle\nabla_{X}(A)^{N}(Y), Z\right\rangle$.

The mean curvature vector $H$ is defined by $H=n^{-1} \operatorname{Tr} B$. A submanifold $M$ is said to be minimal if $H=0$ identically. Moreover $M$ is called a totally geodesic submanifold in $\bar{M}$ if its second fundamental form $B$ is identically zero.

3. $C$-totally real submanifold. Let $\bar{M}$ be a Sasakian manifold with structure tensors $(\phi, \xi, \eta,\langle\rangle$,$) . Then the structure tensors satisfy the following equa-$ tions:

$$
\begin{gathered}
\phi^{2}=-I+\eta \otimes \xi, \quad \phi \xi=0, \quad \eta(\phi X)=0, \quad \eta(\xi)=1, \\
\bar{\nabla}_{X} \xi=\phi X, \quad\left(\bar{\nabla}_{X} \phi\right) Y=\eta(Y) X-\langle X, Y\rangle \xi, \quad X, Y \in \mathfrak{X}(\bar{M}) .
\end{gathered}
$$

\footnotetext{
${ }^{1}$ Manifolds, mappings, metrics, ..., etc. are assumed to be differentiable and of class $C^{\infty}$.
} 
The Sasakian manifold is odd dimensional orientable manifold. When the curvature tensor of a $(2 n+1)$-dimensional Sasakian manifold $\bar{M}$ has the following form

$$
\begin{aligned}
4 \bar{R}(X, Y) Z= & (k+3)(\langle Y, Z\rangle X-\langle X, Z\rangle Y) \\
& +(k-1)\left(\eta(X) \eta(Z) Y-\eta(Y) \eta(Z) X+\langle X, Z\rangle_{\eta}(Y) \xi\right. \\
& \left.-\langle Y, Z\rangle_{\eta}(X) \xi+\langle\phi Y, Z\rangle_{\phi} X+\langle\phi Z, X\rangle_{\phi} Y-2\langle\phi X, Y\rangle_{\phi} Z\right),
\end{aligned}
$$

$\bar{M}$ is called a space of constant $\phi$-holomorphic sectional curvature $k$. In such a space, $k$ is constant if $n>1$. It is well known that an odd dimensional sphere is Sasakian and a Sasakian manifold is a contact manifold.

Let us recall the definition on a $C$-totally real submanifold in a Sasakian manifold.

Let $\bar{M}$ be a $(2 n+1)$-dimensional contact manifold with contact form $\eta$. The Pfaffian equation $\eta=0$ determines in $\bar{M}$ a $2 n$-dimensional distribution. It is called the contact distribution. A submanifold $M$ in $\bar{M}$ is said to be an integral submanifold of the contact distribution if and only if any tangent vector of $M$ belong to the contact distribution. We shall call the integral submanifold $M$ of the contact distribution of a Sasakian manifold a $C$-totally real submanifold. Then we have known $\operatorname{dim} M \leqslant n$, and the following theorem has been proved [9].

THEOREM C. Let $M$ be an $m(m \leqslant n)$ dimensional $C$-totally real submanifold in a Sasakian manifold $\bar{M}^{2 n+1}$ with structure tensors $(\phi, \xi, \eta,\langle\rangle$,$) . Then we have$ the following:

(i) The second fundamental form of $\xi$ direction is identically zero.

(ii) If $X \in \mathfrak{X}(M)$, then $\phi X \in \mathfrak{X}^{\perp}(M)$.

(iii) If $m=n$, then $A^{\phi X}(Y)=A^{\phi Y}(X)$ for $X, Y \in \mathfrak{X}(M)$.

3. Proof of theorem. In the following we assume that $\bar{M}(k)$ is a 5dimensional Sasakian manifold with constant $\phi$-holomorphic sectional curvature and $M$ is a $C$-totally real minimal surface of $\bar{M}(k)$. By the assumptions, we get the Simons' type formula for the second fundamental form $A$ as follows:

$$
\nabla^{2} A=-A \cdot \tilde{A}-A \cdot A+\frac{1}{4}(3 k+5) A,^{2}
$$

where the operators $\tilde{A}$ and $A$ are defined by

$$
\tilde{A}={ }^{t} A \cdot A \quad \text { and } \quad \boldsymbol{A}=\sum_{\alpha=3}^{5} \text { ad } A^{\alpha} \text { ad } A^{\alpha} .
$$

We take a frame $e_{1}, e_{2}$ for $T_{P}(M)$ and a frame $\phi e_{1}, \phi e_{2}, \xi$ for $T_{P}(M)^{\perp}$. Then the second fundamental form can be expressed as

$$
A^{\phi e_{1}}=\left(\begin{array}{cc}
a & 0 \\
0 & -a
\end{array}\right), \quad A^{\phi e_{2}}=\left(\begin{array}{cc}
0 & -a \\
-a & 0
\end{array}\right), \quad A^{\xi}=\left(\begin{array}{ll}
0 & 0 \\
0 & 0
\end{array}\right) .
$$

Therefore, owing to (3.1) and (3.2), we get

\footnotetext{
${ }^{2}$ See [9] or [10].
} 


$$
\frac{1}{2} \Delta\|A\|^{2}=\langle\nabla A, \nabla A\rangle+(3 k+5) a^{2}-24 a^{4} .
$$

By the way, we can easily obtain

$$
\langle\nabla A, \nabla A\rangle=8\|d a\|^{2}+4 a^{2}
$$

because of $\|A\|^{2}=4 a^{2}$. Moreover, by the Gauss equation, it follows that

$$
2 a^{2}=(k+3) / 4-K,
$$

where $K$ denotes the Gauss curvature of $M$, and consequently, it holds that

$$
\Delta\|A\|^{2}=24 a^{2} K+16\|d a\|^{2}
$$

by virtue of (3.3), (3.4) and (3.5). Using (3.6) it is easy to prove

$$
\log \|A\|^{2}=6 K
$$

whenever $\|A\|^{2} \neq 0$. Using the isothermal coordinate and (3.7), it can be proved that either $\|A\|^{2} \equiv 0$ or $\|A\|^{2}$ vanishes only at isolated points.

Let us prove the theorem stated in $\S 1$.

If $M$ has genus zero, then $M$ is totally geodesic by virtue of the GaussBonnet theorem and (3.7).

In the next place, we shall discuss the case of $K \geqslant 0$. Then $M$ is compact or parabolic. Now assume that $M$ is compact. Then by Theorem B, $M$ is totally geodesic if $K>0$. Next we suppose that $M$ is parabolic and noncompact. Then (3.7) implies that $\log \|A\|^{2}$ is subharmonic. On the other hand, we have

$$
\log \|A\|^{2}=\log 4 a^{2}=\log \frac{1}{2}(k+3-4 K) \leqslant \log \frac{1}{2}(k+3) .
$$

Therefore, $\log \|A\|^{2}$ is bounded from above, and this means that $\|A\|^{2}$ is constant on $M$. This completes the proof of the case of $K \geqslant 0$.

In the last place, we consider the case of $K \leqslant 0$. It is possible to choose a system of isothermal coordinates $\left\{x^{1}, x^{2}\right\}$ covering $M$ such that the induced metric tensor $g$ has the following form

$$
g=E\left\{\left(d x^{1}\right)^{2}+\left(d x^{2}\right)^{2}\right\}
$$

Let $g^{*}$ denote the new metric tensor in $M$ given by $g^{*}=\|A\|^{2 / 3} g$. Then, by (3.7), we see that the Gauss curvature $K^{*}$ of $M$ with respect to $g^{*}$ vanishes. Thus, $g^{*}$ is a flat metric in $M$ which is conformally equivalent to $g$. Since $\|A\|^{2 / 3}$ is bounded from below using the assumption, the metric $g^{*}$ is complete, and hence the universal covering surface $\tilde{M}$ of $M$ is conformally equivalent to the Euclidean plane $E^{2}$. The function $\log \|A\|^{2}$ is a globally defined function and is bounded from below by our assumption. Moreover, from (3.7), we see that $\log \|A\|^{2}$ is a superharmonic function. Lifting this to $\tilde{M}$, we obtain a superharmonic function bounded from below on $\tilde{M}$. Therefore $\tilde{M}$ is parabolic, which means that $\log \|A\|^{2}$ is constant. This completes the proof of the case $K \leqslant 0$. Summing up the results obtained above, we get the theorem, and we immediately get the corollary from the theorem. 


\section{REFERENCES}

1. B. Y. Chen, Geometry of submanifolds, Dekker, New York, 1973.

2. B. Y. Chen and K. Ogiue, On totally real submanifolds, Trans. Amer. Math. Soc. 193 (1974), 257-266.

3. C. S. Houh, Some totally real minimal surfaces in $C P^{2}$, Proc. Amer. Math. Soc. 40 (1973), 240-244. MR 47 \#5737.

4. A. Huber, On subharmonic functions and differential geometry in the large, Comment. Math. Helv. 32 (1957), 13-72. MR 20\#970.

5. T. Klotz Milnor and R. Osserman, Complete surfaces in $E^{3}$ with constant mean curvature, Comment. Math. Helv. 41 (1966/67), 313-318. MR 35 \#213.

6. R. Osserman, A survey of minimal surfaces, Van Nostrand Reinhold, New York, 1969. MR 41 \# 934.

7. S. Sasaki, A characterization of contact transformations, Tôhoku Math. J. (2) 16 (1964), 285-290. MR 30 \#355.

8. J. Simons, Minimal varieties in riemannian manifolds, Ann. of Math. (2) 88 (1968), 62-105. MR 38\#1617.

9. S. Yamaguchi and T. Ikawa, On compact minimal C-totally real submanifolds, Tensor (N. S.) 29 (1975), 30-34.

10. S. Yamaguchi, M. Kon and T. Ikawa, On C-totally real submanifolds, J. Differential Geometry (to appear).

11. S. T. Yau, Submanifolds with constant mean curvature. I, Amer. J. Math. 96 (1974), 346-366.

DEPARTMENT OF MATHEMATICS, SCIENCE UNIVERSITY OF TOKYO, TOKYO, JAPAN 